\title{
Decision Model of Equipment Support Command for Large Scale Joint Operations Based on Intuitionistic Fuzzy Reasoning
}

\author{
Huang Xinxin a , Ruan Yongjun and Chen Yukun \\ Ordnance Engineering College, Shijiazhuang 050003, China. \\ aheianzhihou123@126.com
}

Keywords: Intuitionistic fuzzy reasoning; Joint operation; Decision model; Equipment support command.

\begin{abstract}
According to the characteristics of large scale joint operational equipment support command, the factors of equipment support command decision were analyzed. On the basis of fuzzy processing command and decision factors, the intuitionistic fuzzy sets of each decision factors were established, and the corresponding membership and non-membership function were given. Simultaneously, based on intuitionistic fuzzy reasoning, decision model of equipment support command was established, and the inference rules, the intuitionistic fuzzy reasoning algorithm and the de - fuzzy algorithm were presented.
\end{abstract}

\section{Introduction}

The equipment of modern warfare support environment is becoming increasingly complex, command and decision needs to consider all aspects of factors. Therefore, the decision of equipment support has been the research focus of many scholars. With the development of computer technology, the modeling theory and modeling methods are constantly improved, and an increasing number of methods and theories have been introduced into the field [1-5]. Therefore, it is an important content in research on modeling of equipment support command entity that using fuzzy reasoning to make the reasoning and decision of equipment support behavior in Wartime based on incomplete, imprecise or uncertain information.

\section{The theory of intuitionistic fuzzy reasoning}

The concept of intuitionistic fuzzy sets was given by Atanassov in 1986 $6^{[6-8]}$. The difference between the intuitionistic fuzzy sets and the fuzzy sets is mainly that the membership degree of the domain is given, and the non-membership degree is also given in intuitionistic fuzzy sets. The intuitionistic fuzzy set on the domain $X$ is an object of the following form:

$$
A=\left\{\left(x, \mu_{A}(x), \quad \gamma_{A}(x)\right) \mid x \in X\right\}
$$

where $\mu_{A}(x): X \rightarrow[0,1]$ is membership that $X$ belongs to $A, \gamma_{A}(x): X \rightarrow[0,1]$ is membership that $X$ does not belong to $A$, and then:

$$
0 \leq \mu_{A}(x)+\gamma_{A}(x) \leq 1, \quad x \in A
$$

For intuitionistic fuzzy set of $X$, the definition of the intuition index about $x$ is:

$$
\pi_{A}(x)=1-\mu_{A}(x)-\gamma_{A}(x)
$$

Which reflects a measure of the degree of hesitation about $x$ to $A$. When $X$ is a continuous space, the intuitionistic fuzzy set $A$ is:

$$
A=\int_{X}\left(\mu_{A}(x), \quad \gamma_{A}(x)\right) \mathrm{d} x
$$

When $X$ is a discrete space, the intuitionistic fuzzy set $A$ is:

$$
A=\sum_{i=1}^{n}\left(\mu_{A}\left(x_{i}\right), \quad \gamma_{A}\left(x_{i}\right)\right) / x_{i}
$$


Intuitionistic fuzzy set (IFS) is an effective extension and development of Zadeh fuzzy set (ZFS). It adds a new attribute parameters namely non membership function, that is, the membership, non membership and intuitionistic index of IFS can represent three states about support, opposition and neutral. It is more flexible and practical to deal with the uncertainty of fuzzy concepts.

\section{Analysis on the decision factors of equipment support command}

3.1Analysis of enemy's situation and fuzzy processing: The enemy's situation was simplified to enemy fire threat in this paper. The threat of enemy fire was normalized to $x_{1} \in[0,1]$, generally, the greater the $x_{1}$, the higher the degree of fire threat. Here take the enemy fire threat level domain $A \in[0,1]$, and the intuitionistic fuzzy subset are $A_{1} \in\{$ the threat of fire is small $\}$ and $A_{2} \in\{$ the threat of fire is strong\}. Then the intuitionistic fuzzy sets of enemy's situation can be represented as $<x_{1}, \mu_{A_{1}}, \gamma_{A_{1}}>$ and $<x_{1}, \mu_{A_{2}}, \gamma_{A_{2}}>$. Intuitionistic fuzzy membership function and non membership function are shown as (6), (7). At the same time, for the sake of simplicity, suppose $\pi_{A}(x)=0$.

$$
\begin{aligned}
& \mu_{A_{1}}\left(x_{1}\right)=\exp \left(-\frac{x_{1}^{2}}{2 \sigma^{2}}\right) \quad \gamma_{A_{1}}\left(x_{1}\right)=1-\mu_{A_{1}}\left(x_{1}\right) \\
& \mu_{A_{2}}\left(x_{1}\right)=\exp \left(-\frac{\left(x_{1}-1\right)^{2}}{2 \sigma^{2}}\right) \quad \gamma_{A_{2}}\left(x_{1}\right)=1-\mu_{A_{2}}\left(x_{1}\right)
\end{aligned}
$$

here $\sigma=0.2184$.

Analysis of guarantee task and fuzzy processing: In general, the equipment support decision-making strategy is based on the degree of urgency of task to protect. The emergency situation of the security task was normalized to $x_{2} \in[0,1]$. In general, the bigger the $x_{2}$, the higher the degree of emergency is. Due to the different parts of the damage and different degree of damage, different war damage of equipment should spend different time to repair. The expected time to support the equipment was normalized to $x_{3} \in[0,1]$, generally, the larger the $x_{3}$, the longer the expected time to complete the task. Here take security task level domain $B=[0,1]$, the intuitionistic fuzzy subset are $B_{1}=$ \{security task execution temporarily $\}, B_{2}=\{$ security task execution $\}$ and $B_{3}=$ \{support tasks executed immediately\}, Then the intuitionistic fuzzy sets of supporting tasks can be represented as $<\left(x_{2}, x_{3}\right), \mu_{B_{1}}, \gamma_{B_{1}}>$ and $<\left(x_{2}, x_{3}\right), \mu_{B_{3}}, \gamma_{B_{3}}>$. The intuitionistic fuzzy membership function and non membership function are shown as follows:

$$
\begin{gathered}
\mu_{B_{1}}\left(x_{2}, x_{3}\right)=0.5\left[\exp \left(-\frac{x_{2}^{2}}{2 \sigma^{2}}\right)+\exp \left(-\frac{\left(1-x_{3}\right)^{2}}{2 \sigma^{2}}\right)\right] \\
\gamma_{B_{1}}\left(x_{2}, x_{3}\right)=1-\mu_{B_{1}}\left(x_{2}, x_{3}\right) \\
\mu_{B_{2}}\left(x_{2}, x_{3}\right)=0.5\left[\exp \left(-\frac{\left(x_{2}-1 / 2\right)^{2}}{2 \sigma^{2}}\right)+\exp \left(-\frac{\left(x_{3}-1 / 2\right)^{2}}{2 \sigma^{2}}\right)\right] \\
\gamma_{B_{2}}\left(x_{2}, x_{3}\right)=1-\mu_{B_{2}}\left(x_{2}, x_{3}\right) \\
\mu_{B_{3}}\left(x_{2}, x_{3}\right)=0.5\left[\exp \left(-\frac{\left(1-x_{2}\right)^{2}}{2 \sigma^{2}}\right)+\exp \left(-\frac{x_{3}^{2}}{2 \sigma^{2}}\right)\right] \\
\gamma_{B_{3}}\left(x_{2}, x_{3}\right)=1-\mu_{B_{3}}\left(x_{2}, x_{3}\right)
\end{gathered}
$$

here $\sigma=0.1456$.

Analysis of supporting system and fuzzy processing: In the equipment support system of tactical level, the availability of a certain support unit can be expressed by the ratio of the support unit in the 
free state and the total number of the support unit. The availability of a certain security unit is processed to $x_{4} \in[0,1]$, generally, the greater the $x_{4}$, the higher the availability of the equipment support unit.

In the equipment support system of tactical level, the availability of a certain support resources can be expressed by the ratio of the available quantity of this kind of safeguard resources and the quantity of the resource that should be. The availability of a certain support resources is processed to $x_{5} \in[0,1]$, generally, the greater the $x_{5}$, the higher the availability of the equipment support resources. The equipment support defense strength is normalized to $x_{6} \in[0,1]$, generally, the greater the $x_{6}$, the stronger the equipment support defense strength.

Here take the domain of degree of the support system is $C \in[0,1]$, the intuitionistic fuzzy subsets are $C_{1}=\{$ support system cannot be able to perform the tasks $\}, C_{2}=\{$ support system is able to perform the tasks $\},<\left(x_{4}, x_{5}, x_{6}\right), \mu_{C_{1}}, \gamma_{C_{1}}>,<\left(x_{4}, x_{5}, x_{6}\right), \mu_{C_{2}}, \gamma_{C_{2}}>$. The intuitionistic fuzzy membership function and non membership function are shown as follows:

$$
\begin{gathered}
\mu_{C_{1}}\left(x_{4}, x_{5}, x_{6}\right)=0.33\left[\exp \left(-\frac{x_{4}^{2}}{2 \sigma^{2}}\right)+\exp \left(-\frac{x_{5}^{2}}{2 \sigma^{2}}\right)+\exp \left(-\frac{x_{6}^{2}}{2 \sigma^{2}}\right)\right] \\
\gamma_{C_{1}}\left(x_{4}, x_{5}, x_{6}\right)=1-\mu_{C_{1}}\left(x_{4}, x_{5}, x_{6}\right) \\
\mu_{C_{2}}\left(x_{4}, x_{5}, x_{6}\right)=0.33\left[\exp \left(-\frac{\left(1-x_{4}\right)^{2}}{2 \sigma^{2}}\right)+\exp \left(-\frac{\left(1-x_{5}\right)^{2}}{2 \sigma^{2}}\right)+\exp \left(-\frac{\left(1-x_{6}\right)^{2}}{2 \sigma^{2}}\right)\right] \\
\gamma_{C_{2}}\left(x_{4}, x_{5}, x_{6}\right)=1-\mu_{C_{2}}\left(x_{4}, x_{5}, x_{6}\right)
\end{gathered}
$$

here $\sigma=0.2184$.

Conclusion and fuzzy processing: The conclusion of intuitionistic fuzzy reasoning of equipment support command decision is to carry on equipment support or not to carry on equipment support. So the domain of the reasoning result is $Z=[0,1]$, take its intuitionistic fuzzy subset are $Z_{1}=\{$ to carry on the equipment support $\}, Z_{2}=\{$ does not carry on the equipment safeguard $\}$. The intuitionistic fuzzy subsets of the enemy can be expressed as $<z_{1}, \mu_{Z_{2}}, \gamma_{Z_{2}}>$ and $<z_{2}, \mu_{Z_{2}}, \gamma_{Z_{2}}>$.

Rules of fuzzy inference: As the input parameters of the fuzzy inference system, the number of subsets of decision factors $A 、 B 、 C$ are $N_{A}=2 、 N_{B}=3 、 N_{C}=2$. It can be calculated that the number of rules in the inference system is 12 . The rule $R(k)$ can be expressed as: $R(k):$ If $A$ is $A_{a i}$ and $B$ is $B_{b i}$ and $C$ is $C_{c i}$, then $Z$ is $Z_{j}: C_{F}(k)$. Of which: (1) $A 、 B 、 C$ are input fuzzy variables of system, and as linguistic terms of fuzzy preconditions, $A_{a i} 、 B_{b i} 、 C_{c i}$ are intuitionistic fuzzy sets in each domain, that are $<a, \mu_{a_{i}}, \gamma_{a_{i}}>, a \in A,<b, \mu_{b_{i}}, \gamma_{b_{i}}>, b=\left(x_{2}, x_{3}\right) \in B,<c, \mu_{c_{i}}, \gamma_{c_{i}}>$, $c=\left(x_{4}, x_{5}, x_{6}\right) \in C . Z$ is the output fuzzy variables of the system and also the conclusion part of rules, $Z_{j}$ is the intuition fuzzy subsets of output domain, $<z, \mu_{j}, \gamma_{j}>, z \in Z, a_{i}=1,2, b_{i}=1,2,3, c_{i}=1,2$, $j=1,2$. (2) $C_{F}(k)$ is the credibility of $R(k)$, given by the military experts in the field, reflects the degree of confidence about the uncertainty knowledge based on their professional knowledge and practical experience.

Algorithm of fuzzy inference synthesis: This paper adopts the synthetic operation $\wedge-\vee, R(k)$ should satisfy the following equation: 


$$
\begin{array}{r}
\mu_{R(k)}(a, b, c ; z)=\mu_{A_{a i}}(a) \wedge \mu_{B_{b i}}(b) \wedge \mu_{C_{c i}}(c) \wedge \mu_{Z_{j}}(z) \wedge C_{F}(k)= \\
\mu_{A_{a i}}\left(x_{1}\right) \wedge \mu_{B_{b i}}\left(x_{2}, x_{3}\right) \wedge \mu_{C_{c i}}\left(x_{4}, x_{5}, x_{6}\right) \wedge \mu_{Z_{j}}(z) \\
\gamma_{R(k)}(a, b, c ; g)= \\
\gamma_{A_{a i}}(a) \vee \gamma_{B_{b i}}(b) \vee \gamma_{C_{c i}}(c) \vee \gamma_{Z_{j}}(z) \vee C_{F}(k)= \\
\gamma_{A_{a i}}\left(x_{1}\right) \vee \gamma_{B_{b i}}\left(x_{2}, x_{3}\right) \vee \gamma_{C_{c i}}\left(x_{4}, x_{5}, x_{6}\right) \vee \gamma_{Z_{j}}(z)
\end{array}
$$

$\forall a \in A, \quad \forall b \in B, \quad \forall c \in C, \quad \forall z \in Z, \quad k=1,2 \ldots, 12$

When inputs are $A^{\prime} 、 B^{\prime} 、 C^{\prime}$, we can know the result of the inference is $Z_{k}{ }^{\prime}=\left(A^{\prime} \times B^{\prime} \times C^{\prime}\right) \bullet R(k)$, which satisfy:

$$
\begin{gathered}
\mu_{Z_{k}^{\prime}}(a, b, c ; z)=\underset{a, b, c}{\vee}\left(\mu_{A^{\prime}}(a) \wedge \mu_{B^{\prime}}(b) \wedge \mu_{C^{\prime}}(c) \wedge \mu_{R(k)}(a, b, c ; z)\right) \\
\gamma_{Z_{k}^{\prime}}(a, b, c ; z)=\underset{a, b, c}{\wedge}\left(\gamma_{A}(a) \vee \gamma_{B}(b) \vee \gamma_{C}(c) \vee \gamma_{R(k)}(a, b, c ; z)\right)
\end{gathered}
$$

Synthesis each inference rules, the membership degree and non membership degree of intuitionistic fuzzy sets $Z^{\prime}$ were obtained:

$$
\mu_{Z^{\prime}}(z)=\underset{k}{\vee} \mu_{Z_{k}^{\prime}}(z) \quad \gamma_{Z^{\prime}}(z)=\hat{k}_{k} \gamma_{Z_{k}^{\prime}}(z)
$$

Algorithm to remove fuzziness: Intuitionistic fuzzy set $Z^{\prime}$ was used to reason fuzzily about equipment support command decision-making. The fuzziness of reasoning result should be removed. In this paper, the maximum truth value method was used to draw the final conclusion. The true value of the intuitionistic fuzzy set $V$ on the domain $U$ is:

$$
T_{V}(u)=\alpha \times \mu_{V}(u)+\beta \times \pi_{V}(u), \quad u \in U
$$

\section{Conclusion}

There are a lot of fuzzy uncertainty factors in the equipment support decision information. In this paper, the theory of threat assessment is used to apply intuitionistic fuzzy reasoning method for command decision making. It can effectively deal with the uncertainty problems caused by the fuzziness of knowledge. In dealing with the fuzzy concept of the equipment support command decision, it is practical, flexible and targeted, and also has capacity of imitating human experts. This method provides a new idea and method for the modeling of equipment support command and decision, and it certainly make foundation for the future research.

\section{References}

[1] TAN Ya-Xin, WANG Cheng, FAN Rui. Study of Command Decision Model Based on Prearranged Command Regulation[J]. Journal of System Simulation. 2008,20(14):3820-3824.

[2] Li Yi-long, Yu Tao. Rule-Based Command and Control in Sea Battles[J]. Command Information System and Technology.2011,2(5):47-50.

[3] CHEN Hai, HU Jian-wan. Research on the Simulation of Command Control System based on Petri Net[J]. Fire Control and Command Control.2009,34(6):65-67.

[4] YI Hong-bing, ZHANG Chun-run, LIU Zeng-yong, et al. Research on Application of Fuzzy Petri-Net for Equipment Support Command Decision[J]. Command Control and Simulation. 2008,30(4):99-102.

[5] LI Bi-cheng, WANG Jin, LIN Chen. Method of online public opinions prewaring based on intuitionistic fuzzy reasoning[J]. Application Research of Computers.2010, 27(9):3312-3315.

[6] CHEN Dong-feng, LEI Ying-jie. An Intuitionistic Fuzzy Inference Method for Threat Assessment [J]. Command Control and Simulation.2006,28(4):21-23. 